\author{
ANNALES \\ POLONICI MATHEMATICI \\ $\mathrm{XXV}$ (1972)
}

\title{
The mean surface area of the boxes circumscribed about a convex body
}

\author{
by ROLF SCHNEIDER (Franlefurt am Main)
}

A convex polytope is said to be circumseribed about a convex body if and only if each of its facets is contained in a support plane of the body.

Radziszewslri [4] has proved the following theorem:

In the plane, let $\mathbb{K}$ be a convex body of area $A(K)$, and let $B(K, \varphi)$ denote the area of the rectangle oiroumscribed about $K$ suoh that one of its sides forms an angle $\varphi$ with a fixed direction. Then the inequality

$$
(2 \pi)^{-1} \int_{0}^{2 \pi} B(K, \varphi) d \varphi \geqslant 4 \pi^{-1} A(K)
$$

is valid, where equality holds if and only if $K$ is a oircular diso.

This inequality was recently rediscovered by Chernoff [2]; see also Feil [3] for some generalizations and consequences.

The left-hand side of (1) may be interpreted as the mean value of the areas of all the rectangles circumscribed about $K$. In the following we shall prove an analogous inequality in three-dimensional space. The method will bo similar to that of Chernoff. It seems that Radziszewski's proof does not immediately extend to higher dimensions, since such an extension would require an, apparently unknown, improved version of a Minkowskian inequality.

Let $S^{2}=\left\{u \in E^{3} \mid\langle u, u\rangle=1\right\}$ be the unit sphere of three-dimensional Euclidean space $E^{3}\left(\langle\right.$,$\left.\rangle denotes the scalar product in E^{3}\right)$; let $S O(3)$ denote the rotation group acting on $\mathcal{S}^{2}$. If $\left(u_{1}, u_{2}, u_{3}\right)$ is a fixed orthonormal frame in $E^{3}$ and if $\delta \epsilon S O(3)$ is a rotation, then there is a unique box (= rectangular parallelepiped) circumscribed about a given convex body $\pi \subset E^{3}$ such that the exterior unit normal vectors of the box are $\pm \delta u_{1}, \pm \delta u_{2}, \pm \delta u_{3}$. Let $B(K, \delta)$ denote the surface area of this box. Then the mean value of the 
surface areas of the boxes circumscribed about $K$ is defined in a natural way by

$$
\breve{B}(\mathbb{K})=\int_{S O(9)} B(I, \delta) d \mu(\delta),
$$

where $\mu$ denotes the normalized Haar measure on the.group $S O(3)$.

THEOREM. If $K \subset \mathbb{B}^{3}$ is a oonvex body with surfaes area $A(K)$, then

$$
\bar{B}(I) \geqslant 6 \pi^{-1} \cdot A(K) \text {. }
$$

Equality holds if and only if $\mathbb{K}$ is a sphorioal ball.

Proof. Let $p(K, \imath)$.denote the support function of $K$, restricted to $S^{2}$, that is $p(K, u)=\sup \{\langle\infty, u\rangle \mid w \in K\}$ for $u \in S^{z}$. Let $Y_{m 1}, \ldots, Y_{m, 2 m-1-1}$ be an orthonormal basis of the real vector space (with the usual scalar product) of spherical surface harmonics of degree $m$ on $S^{z}(m=0,1,2, \ldots)$. We will first assume that the support function of $K$ is differentiable sufficiently often, so that it can be represented as an absolutely and uniformly convergent series of spherical harmonics

$$
p(\Pi, u)=\sum_{m=0}^{\infty} \sum_{i=1}^{2 m+1} a_{m i} Y_{m i}(u)^{\prime}
$$

where

$$
a_{m i}=\int_{S^{2}} p(K, u) Y_{m i}(u) d \omega(u)
$$

( $\omega$ denotes the Lebesgue measure on $S^{2}$ ). It is well known (see Blaschle [1], p. 108-110) that the surface area of $K$ is now expressed by

$$
A(K)=a_{01}^{2}-\frac{1}{2} \sum_{m=2}^{\infty}(m-1)(m+2) \sum_{i=1}^{2 m+1} a_{m i}^{2} .
$$

The functional $B(K, \delta)$ is given by where

$$
B(K, \delta)=2\left[b\left(\delta u_{1}\right) b\left(\delta u_{2}\right)+b\left(\delta u_{2}\right) b\left(\delta u_{3}\right)+b\left(\delta u_{3}\right) b\left(\delta u_{1}\right)\right],
$$

$$
b(u)=p(K, u)+p(K,-u) .
$$

By the invariance of the Haar measure we obtain

which leads to

$$
\bar{B}(K)=6 \int_{S O(3)} b\left(\delta u_{1}\right) b\left(\delta u_{2}\right) d \mu(\delta),
$$

$$
\bar{B}(K)=24 \sum_{\substack{m=0 \\ 2 \mid m}}^{\infty} \sum_{\substack{k=0 \\ 2 \mid k}}^{\infty} \sum_{i=1}^{2 m+1} \sum_{j=1}^{2 k+1} a_{m i} a_{k j} \int_{S O(9)} Y_{m i}\left(\delta u_{1}\right) Y_{l j}\left(\delta u_{2}\right) d \mu(\delta)
$$


(observe that $Y_{m i}(u)=(-1)^{m} \cdot Y_{m i}(-u)$ ). Now for any continuous function $f$ on $S^{2}$ and any two vectors $u, v \in \mathbb{S}^{2}$ the formula

$$
\int_{S O(3)} f(\delta u) Y_{m i}(\delta v) d \mu(\delta)=(4 \pi)^{-1} \int_{S^{2}} f Y_{m i} d \omega P_{m}(\langle u, v\rangle)
$$

is valid, where $P_{m}$ is the Legendre polynomial of degree $m$. This follows from the fact that the left-hand side is, as a function of $v$ alone, a spherical harmonic of degree $m$, which, by the invariance of the Haar measure, turns out to depend only on $\langle u, v\rangle$; hence it must be a constant multiple of $P_{m}(\langle u, v\rangle)$. The factor is then easily determined. (The complete argument; may be found in [5], (3.3).) Since $\left\langle u_{1}, u_{2}\right\rangle=0$ and since the system $\left\{Y_{m i}\right\}$ is orthonormal, this yields

$$
\bar{B}(K)=6 \pi^{-1} \sum_{m=0}^{\infty} P_{m}(0) \sum_{i=1}^{2 m+1} a_{m i}^{2}
$$

(we have $P_{m}(0)=0$ for odd $m$ ). In view of $(3)$ we arrive at

$$
\bar{B}(K)-6 \pi^{-1} A(K)=6 \pi^{-1} \sum_{m=2}^{\infty}\left[P_{m}(0)+\frac{1}{2}(m-1)(m+2)\right] \sum_{i=1}^{2 m+1} a_{m i}^{2} \geqslant 0,
$$

since $P_{0}(0)=1$ and $\left|P_{m}(0)\right| \leqslant 1$. A standard approximation argument now shows that inequality (2) holds for arbitrary convex bodies $K$.

Suppose that for a convex body $K$ the equality sign holds in (2). For sufficiently smooth $K$ we have, according to (4),

$$
\begin{aligned}
& \bar{B}(K)-6 \pi^{-1} A(K) \\
& \quad \geqslant 6 \pi^{-1}\left[P_{m}(0)+\frac{1}{2}(m-1)(m+2)\right] \sum_{i=1}^{2 m+1}\left\{\int_{S^{2}} p(K, u) Y_{m i}(u) d \omega(u)\right\}^{2}
\end{aligned}
$$

for $m=2,3$, By approximation, this holds for general $K$. Since we have assumed the left-hand side to be zero, it follows that

$$
\int_{S^{2}} p(K, u) Y_{m i}(u) d \omega(u)=0
$$

for $i=1,2, \ldots, 2 m+1$ and for $m=2,3$, From the completeness of the system of spherical harmonics we conclude that

$$
p(K, \cdot)=Y_{0}+Y_{1}
$$

where $Y_{k}$ is a spherical hammonic of degree $k(k=0,1)$. But $Y_{0}$ is constiant and $Y_{1}(u)=\langle c, u\rangle$ with some constant vector $c$; hence $K$ is a spherical ball.

Remarks. The preceding theorem and its proof extend to arbitrary dimensions $n \geqslant 2$ provided the surface area functional is replaced by the 
quermass integral $W_{n-2}$. It is, however, an open question whether an analogous result holds for each of the quermass integrals $W_{k}, 0 \leqslant k \leqslant n-3$, especially for the volume $W_{0}$. (For $W_{n-1}$, which is essentially the mean width, there is an identity instead of an inequality.)

Results analogous to the above theorem can be proved if the boxes are replaced by other suitable circumscribed polytopes, for instance by simplices which are similar to a given simplex.

\section{Reforences}

[1] W. Blaschke, Kreis und Kugsl, 2nd ed., Borlin 1956.

[2] P. R. Chern off, $\Delta n$ area-width inoquality for oonvex ourves, Amor. Math. Monthly $76(1969), p .34 .35$.

[3] E. Heil, Eine Versohdrfung der Bieberbachsohen Ungleiohung und einige andere Abschatzungen für ebene lonvexe Bereiche, Elem. Math. (to appear).

[4] K. Radziszewski, Sur une fonctionnelle définio sur les ovales, Ann. Univ. Mariae Curie-Sklodowska, Sect. A., 10 (1950), p. 57-69.

[6] R. Sohneider, Gleitkörper in konvexen Polytopon, J. roine angow. Math. 248 (1971), p. $193-200$.

MATHIMLATISOHES GEMINAR DIER UNIVERSI'A'T FRANKEURT AM MAIN (BRD)
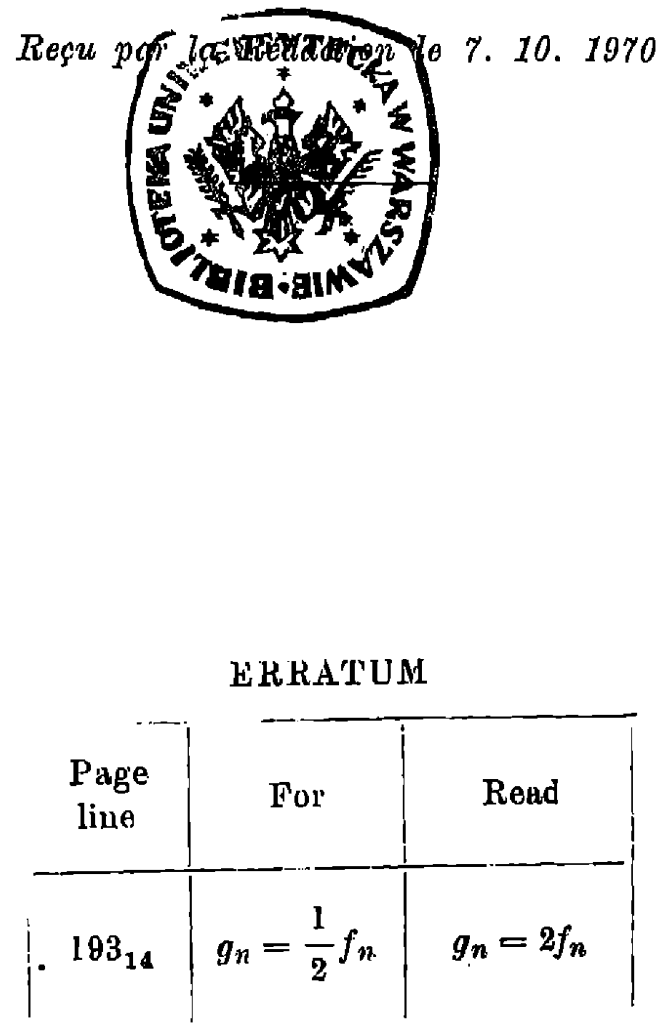

Annales Polonial Mathematlei XXV 